\title{
String topology for spheres
}

\author{
Luc Menichi*
}

With an appendix by Gerald Gaudens and Luc Menichi

Dedicated to Jean-Claude Thomas, on the occasion of his 60th birthday

\begin{abstract}
Let $M$ be a compact oriented $d$-dimensional smooth manifold. Chas and Sullivan have defined a structure of Batalin-Vilkovisky algebra on $\mathbb{H}_{*}(L M)$. Extending work of Cohen, Jones and Yan, we compute this Batalin-Vilkovisky algebra structure when $M$ is a sphere $S^{d}, d \geq 1$. In particular, we show that $\mathbb{H}_{*}\left(L S^{2} ; \mathbb{F}_{2}\right)$ and the Hochschild cohomology $H H^{*}\left(H^{*}\left(S^{2}\right) ; H^{*}\left(S^{2}\right)\right)$ are surprisingly not isomorphic as Batalin-Vilkovisky algebras, although we prove that, as expected, the underlying Gerstenhaber algebras are isomorphic. The proof requires the knowledge of the Batalin-Vilkovisky algebra $H_{*}\left(\Omega^{2} S^{3} ; \mathbb{F}_{2}\right)$ that we compute in the Appendix.
\end{abstract}

Mathematics Subject Classification (2000). 55P35, 16E40, 55P62, 57T30, 55U10.

Keywords. String topology, Batalin-Vilkovisky algebra, Gerstenhaber algebra, Hochschild cohomology, free loop space.

\section{Introduction}

Let $M$ be a compact oriented $d$-dimensional smooth manifold. Denote by $L M:=$ $\operatorname{map}\left(S^{1}, M\right)$ the free loop space on $M$. In 1999, Chas and Sullivan [2] have shown that the shifted free loop homology $\mathbb{H}_{*}(L M):=H_{*+d}(L M)$ has a structure of Batalin-Vilkovisky algebra (Definition 5). In particular, they showed that $\mathbb{H}_{*}(L M)$ is a Gerstenhaber algebra (Definition 8). This Batalin-Vilkovisky algebra has been computed when $M$ is a complex Stiefel manifold [25] and very recently over $\mathbb{Q}$ when $M$ is a $K(\pi, 1)$ [28]. In this paper, we compute the Batalin-Vilkovisky algebra $\mathbb{H}_{*}(L M ; \mathbb{k})$ when $M$ is a sphere $S^{n}, n \geq 1$ over any commutative ring $\mathbb{k}$ (Theorems 10, 16, 17, 24 and 25).

\footnotetext{
${ }^{*}$ The author was partially supported by the Mathematics Research Center of Stanford University.
} 
In fact, few calculations of this Batalin-Vilkovisky algebra structure or even of the underlying Gerstenhaber algebra structure have been done because the following conjecture has not yet been proved.

Conjecture 1 (due to [2, "dictionary" p. 5] or [7]?). If $M$ is simply connected then there is an isomorphism of Gerstenhaber algebras $\mathbb{H}_{*}(L M) \cong H H^{*}\left(S^{*}(M) ; S^{*}(M)\right)$ between the free loop space homology and the Hochschild cohomology of the algebra of singular cochains on $M$.

In [7], [5], Cohen and Jones proved that there is an isomorphism of graded algebras over any field

$$
\mathbb{H}_{*}(L M) \cong H H^{*}\left(S^{*}(M) ; S^{*}(M)\right) .
$$

Over the reals or over the rationals, two proofs of this isomorphism of graded algebras have been given by Merkulov [23] and Félix, Thomas, Vigué-Poirrier [11]. Motivated by this conjecture, Westerland [30] has computed the Gerstenhaber algebra $H H^{*}\left(S^{*}\left(M ; \mathbb{F}_{2}\right) ; S^{*}\left(M ; \mathbb{F}_{2}\right)\right)$ when $M$ is a sphere or a projective space.

What about the Batalin-Vilkovisky algebra structure?

Suppose that $M$ is formal over a field, then since the Gerstenhaber algebra structure on Hochschild cohomology is preserves by quasi-isomorphism of algebras [10, Theorem 3], we obtain an isomorphism of Gerstenhaber algebras

$$
H H^{*}\left(S^{*}(M) ; S^{*}(M)\right) \cong H H^{*}\left(H^{*}(M) ; H^{*}(M)\right) .
$$

Poincaré duality induces an isomorphism of $H^{*}(M)$-modules

$$
\Theta: H^{*}(M) \rightarrow H^{*}(M)^{\vee} \text {. }
$$

Therefore, we obtain the isomorphism

$$
H H^{*}\left(H^{*}(M) ; H^{*}(M)\right) \cong H H^{*}\left(H^{*}(M) ; H^{*}(M)^{\vee}\right)
$$

and the Gerstenhaber algebra structure on $H H^{*}\left(H^{*}(M) ; H^{*}(M)\right)$ extends to a Batalin-Vilkovisky algebra [26], [22], [19] (See above Proposition 20 for details). This Batalin-Vilkovisky algebra structure is further extended in [27], [9], [20], [21] to a richer algebraic structure. It is natural to conjecture that this Batalin-Vilkovisky algebra on $H H^{*}\left(H^{*}(M) ; H^{*}(M)\right)$ is isomorphic to the Batalin-Vilkovisky algebra $\mathbb{H}_{*}(L M)$. We show (Corollary 30 ) that this is not the case over $\mathbb{F}_{2}$ when $M$ is the sphere $S^{2}$. See [6, Comments 2, Chapter 1] or the papers of Tradler and Zeinalian [26], [27] for a related conjecture when $M$ is not assumed to be necessarily formal. On the contrary, we prove (Corollary 23) that the above conjecture is satisfied for $M=S^{2}$ over $\mathbb{F}_{2}$.

Acknowledgment. We wish to thank Ralph Cohen and Stanford Mathematics Department for providing a friendly atmosphere during my six months of "delegation CNRS". We would like also to thank Yves Félix for a discussion simplifying the proof of Theorem 10. 


\section{The Batalin-Vilkovisky algebra structure on $\mathbb{H}_{*}(L M)$}

We recall here the definition of the Batalin-Vilkovisky algebra on $\mathbb{H}_{*}(L M ; \mathbb{k})$ given by Chas and Sullivan [2] over any commutative ring $\mathbb{k}$ and deduce that this BatalinVilkovisky algebra $\mathbb{H}_{*}(L M ; \mathbb{k})$ behaves well with respect to change of rings.

We first recall the definition of the loop product following Cohen and Jones [7], [6]. Let $M$ be a closed oriented smooth manifold of dimension $d$. The inclusion $e: \operatorname{map}\left(S^{1} \vee S^{1}, M\right) \hookrightarrow L M \times L M$ can be viewed as a codimension $d$ embedding between infinite dimension manifolds [24, Proposition 5.3]. Denote by $v$ its normal bundle. Let $\tau_{e}: L M \times L M \rightarrow \operatorname{map}\left(S^{1} \vee S^{1}, M\right)^{v}$ its Thom-Pontryagin collapse map. Recall that the umkehr (Gysin) map $e_{\text {! }}$ is the composite of $\tau_{e}$ and the Thom isomorphism:

$$
\begin{aligned}
& H_{*}(L M \times L M ; \mathbb{k}) \stackrel{H_{*}\left(\tau_{e} ; \mathrm{k}\right)}{\longrightarrow} H_{*}\left(\operatorname{map}\left(S^{1} \vee S^{1}, M\right)^{v} ; \mathbb{k}\right) \\
& \stackrel{\cap u_{\mathrm{k}}}{\cong} H_{*-d}\left(\operatorname{map}\left(S^{1} \vee S^{1}, M\right) ; \mathbb{k}\right) .
\end{aligned}
$$

The Thom isomorphism is given by taking a relative cap product $\cap$ with a Thom class for $v, u_{\mathbb{k}} \in H^{d}\left(\operatorname{map}\left(S^{1} \vee S^{1}, M\right)^{v} ; \mathbb{k}\right)$. A Thom class with coefficients in $\mathbb{Z}, u_{\mathbb{Z}}$, gives rise to a Thom class $u_{\mathbb{k}}$ with coefficients in $\mathbb{k}$, under the morphism

$$
H^{d}\left(\operatorname{map}\left(S^{1} \vee S^{1}, M\right) ; \mathbb{Z}\right) \rightarrow H^{d}\left(\operatorname{map}\left(S^{1} \vee S^{1}, M\right) ; \mathbb{k}\right)
$$

induced by the ring homomorphism $\mathbb{Z} \rightarrow \mathbb{k}[16, \mathrm{p}$. 441]. So we have the commutative diagram

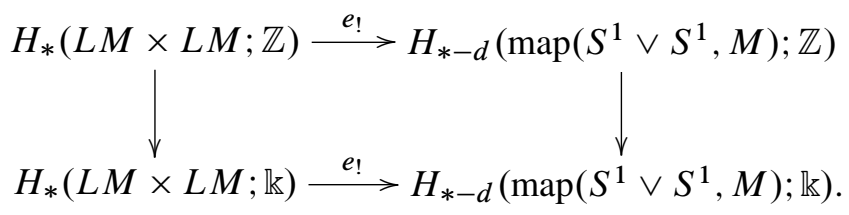

Let $\gamma: \operatorname{map}\left(S^{1} \vee S^{1}, M\right) \rightarrow L M$ be the map obtained by composing loops. The loop product is the composite

$$
\begin{aligned}
H_{*}(L M ; \mathbb{k}) & \otimes H_{*}(L M ; \mathbb{k}) \rightarrow H_{*}(L M \times L M ; \mathbb{k}) \\
& \stackrel{e_{!}}{\longrightarrow} H_{*-d}\left(\operatorname{map}\left(S^{1} \vee S^{1}, M\right) ; \mathbb{k}\right) \stackrel{H_{*-d}(\gamma ; \mathbb{k})}{\longrightarrow} H_{*-d}(L M ; \mathbb{k}) .
\end{aligned}
$$

So clearly, we have proved

Lemma 3. The morphism of abelian groups $\mathbb{H}_{*}(L M ; \mathbb{Z}) \rightarrow \mathbb{H}_{*}(L M ; \mathbb{k})$ induced by $\mathbb{Z} \rightarrow \mathbb{k}$ is a morphism of graded rings. 
Suppose that the circle $S^{1}$ acts on a topological space $X$. Then we have an action of the algebra $H_{*}\left(S^{1}\right)$ on $H_{*}(X)$,

$$
H_{*}\left(S^{1}\right) \otimes H_{*}(X) \rightarrow H_{*}(X) .
$$

Denote by $\left[S^{1}\right]$ the fundamental class of the circle. Then we define an operator of degree $1, \Delta: H_{*}(X ; \mathbb{k}) \rightarrow H_{*+1}(X ; \mathbb{k})$, which sends $x$ to the image of $\left[S^{1}\right] \otimes x$ under the action. Since $\left[S^{1}\right]^{2}=0, \Delta \circ \Delta=0$. The following lemma is obvious.

Lemma 4. Let $X$ be a $S^{1}$-space. We have the commutative diagram

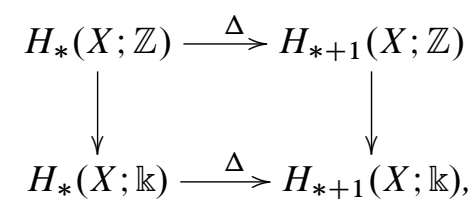

where the vertical maps are induced by the ring homomorphism $\mathbb{Z} \rightarrow \mathbb{k}$.

The circle $S^{1}$ acts on the free loop space on $M$ by rotating the loops. Therefore we have a operator $\Delta$ on $\mathbb{H}_{*}(L M)$. Chas and Sullivan [2] have shown that $\mathbb{H}_{*}(L M)$ equipped with the loop product and the $\Delta$-operator, is a Batalin-Vilkovisky algebra.

Definition 5. A Batalin-Vilkovisky algebra is a commutative graded algebra $A$ equipped with an operator $\Delta: A \rightarrow A$ of degree 1 such that $\Delta \circ \Delta=0$ and

$$
\begin{aligned}
\Delta(a b c)= & \Delta(a b) c+(-1)^{|a|} a \Delta(b c)+(-1)^{(|a|-1)|b|} b \Delta(a c) \\
& -(\Delta a) b c-(-1)^{|a|} a(\Delta b) c-(-1)^{|a|+|b|} a b(\Delta c) .
\end{aligned}
$$

Consider the bracket $\{$,$\} of degree +1$ defined by

$$
\{a, b\}=(-1)^{|a|}\left(\Delta(a b)-(\Delta a) b-(-1)^{|a|} a(\Delta b)\right)
$$

for any $a, b \in A$. (6) is equivalent to the following relation called the Poisson relation:

$$
\{a, b c\}=\{a, b\} c+(-1)^{(|a|+1)|b|} b\{a, c\} .
$$

Getzler [14, Proposition 1.2] has shown that $\{$,$\} is a Lie bracket and therefore that a$ Batalin-Vilkovisky algebra is a Gerstenhaber algebra.

Definition 8. A Gerstenhaber algebra is a commutative graded algebra $A$ equipped with a linear map $\{-,-\}: A \otimes A G \rightarrow A$ of degree 1 such that:

a) the bracket $\{-,-\}$ gives to $A$ a structure of a graded Lie algebra of degree 1 . This means that for each $a, b$ and $c \in A$,

$$
\{a, b\}=-(-1)^{(|a|+1)(|b|+1)}\{b, a\},
$$


and

$$
\{a,\{b, c\}\}=\{\{a, b\}, c\}+(-1)^{(|a|+1)(|b|+1)}\{b,\{a, c\}\} .
$$

b) The product and the Lie bracket satisfy the Poisson relation (7).

Using Lemma 3 and Lemma 4, we deduce

Proposition 9. The $\mathbb{k}$-linear map

$$
\mathbb{H}_{*}(L M ; \mathbb{Z}) \otimes_{\mathbb{Z}} \mathbb{k} \hookrightarrow \mathbb{H}_{*}(L M ; \mathbb{k})
$$

is an inclusion of Batalin-Vilkovisky algebras.

In particular, by the universal coefficient theorem,

$$
\mathbb{H}_{*}(L M ; \mathbb{Z}) \otimes_{\mathbb{Z}} \mathbb{Q} \cong \mathbb{H}_{*}(L M ; \mathbb{Q}) .
$$

More generally, this proposition tells us that if $\operatorname{Tor}^{\mathbb{Z}}\left(\mathbb{H}_{*}(L M ; \mathbb{Z}), \mathbb{k}\right)=0$ then the Batalin-Vilkovisky algebra $\mathbb{H}_{*}(L M ; \mathbb{Z})$ determines the Batalin-Vilkovisky algebra $\mathbb{H}_{*}(L M ; \mathbb{k})$.

\section{The circle and an useful lemma}

In this section, we compute the structure of the Batalin-Vilkovisky algebra on the homology of the free loop space on the circle $S^{1}$ using a lemma which gives information on the image of $\Delta$ on elements of lower degree in $H_{*}(L M)$.

Theorem 10. As a Batalin-Vilkovisky algebra, the homology of the free loop space on the circle is given by

$$
\mathbb{H}_{*}\left(L S^{1} ; \mathbb{k}\right) \cong \mathbb{k}[\mathbb{Z}] \otimes \Lambda a_{-1} .
$$

Denote by $x$ a generator of $\mathbb{Z}$. The operator $\Delta$ is

$$
\Delta\left(x^{i} \otimes a_{-1}\right)=i\left(x^{i} \otimes 1\right), \quad \Delta\left(x^{i} \otimes 1\right)=0
$$

for all $i \in \mathbb{Z}$.

Let $X$ be a pointed topological space. Consider the free loop fibration $\Omega X \stackrel{j}{\hookrightarrow}$ $L X \stackrel{\mathrm{ev}}{\longrightarrow} X$. Denote by $\operatorname{hur}_{X}: \pi_{n}(X) \rightarrow H_{n}(X)$ the Hurewicz map.

Lemma 11. Let $n \in \mathbb{N}$. Let $f \in \pi_{n+1}(X)$. Denote by $\tilde{f} \in \pi_{n}(\Omega X)$ the adjoint of $f$. Then

$$
\left(H_{*}(\mathrm{ev}) \circ \Delta \circ H_{*}(j) \circ \operatorname{hur}_{\Omega X}\right)(\tilde{f})=\operatorname{hur}_{X}(f) .
$$


Proof. Take in homology the image of $\left[S^{1}\right] \otimes\left[S^{n}\right]$ in the following commutative diagram:

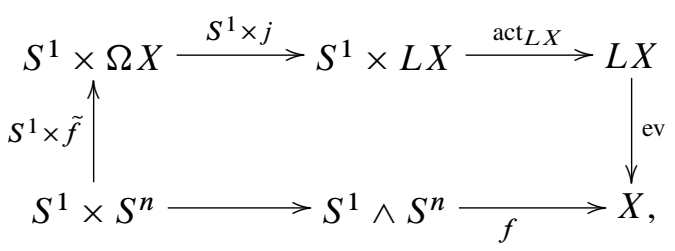

where $\operatorname{act}_{L X}: S^{1} \times L X \rightarrow L X$ is the action of the circle on $L X$.

Proof of Theorem 10. More generally, let $G$ be a compact Lie group. Consider the homeomorphism $\Theta_{G}: \Omega G \times G \stackrel{\cong}{\longrightarrow} L G$ which sends the couple $(w, g)$ to the free loop $t \mapsto w(t) g$. In fact, $\Theta_{G}$ is an isomorphism of fiberwise monoids. Therefore by [15, Part 2 of Theorem 8.2],

$$
\mathbb{H}_{*}\left(\Theta_{G}\right): H_{*}(\Omega G) \otimes \mathbb{H}_{*}(G) \rightarrow \mathbb{H}_{*}(L G)
$$

is a morphism of graded algebras. Since $H_{*}\left(S^{1}\right)$ has no torsion,

$$
\mathbb{H}_{*}\left(\Theta_{S^{1}}\right): H_{*}\left(\Omega S^{1}\right) \otimes \mathbb{H}_{*}\left(S^{1}\right) \cong \mathbb{H}_{*}\left(L S^{1}\right)
$$

is an isomorphism of algebras. Since $\Delta$ preserves path-connected components,

$$
\Delta\left(x^{i} \otimes a_{-1}\right)=\alpha\left(x^{i} \otimes 1\right)
$$

where $\alpha \in \mathbb{k}$. Denote by $\varepsilon_{\mathbb{k}[\mathbb{Z}]}$ the canonical augmentation of the group ring $\mathbb{k}[\mathbb{Z}]$. Since $H_{*}\left(\mathrm{ev} \circ \Theta_{S^{1}}\right)=\varepsilon_{\mathbb{k}[\mathbb{Z}]} \otimes H_{*}\left(S^{1}\right)$,

$$
\left(H_{*}(\mathrm{ev}) \circ \Delta\right)\left(x^{i} \otimes a_{-1}\right)=\alpha 1 .
$$

On the other hand, applying Lemma 11 to the degree $i$ map $S^{1} \rightarrow S^{1}$, we obtain that $\left(H_{*}(\mathrm{ev}) \circ \Delta \circ H_{*}(j)\right)\left(x^{i}\right)=i 1$. Therefore $\alpha=i$.

\section{Computations using Hochschild homology}

In this section, we compute the Batalin-Vilkovisky algebra $\mathbb{H}_{*}\left(L S^{n}\right), n \geq 2$, using the following elementary technique:

The algebra structure has been computed by Cohen, Jones and Yan using the Serre spectral sequence [8]. On the other hand, the action of $H_{*}\left(S^{1}\right)$ on $H_{*}\left(L S^{n}\right)$ can be computed using Hochschild homology. Using the compatibility between the product and $\Delta$, we determine the Batalin-Vilkovisky algebra $\mathbb{H}_{*}\left(L S^{n}\right)$ up to isomorphism. This elementary technique will fail for $\mathbb{H}_{*}\left(L S^{2}\right)$. 
Let $A$ be an augmented differential graded algebra. Denote by $s \bar{A}$ the suspension of the augmentation ideal $\bar{A},(s \bar{A})_{i}=\bar{A}_{i-1}$. Let $d_{1}$ be the differential on the tensor product of complexes $A \otimes T(s \bar{A})$. The (normalized) Hochschild chain complex, denoted $\mathcal{C}_{*}(A ; A)$, is the complex $\left(A \otimes T(s \bar{A}), d_{1}+d_{2}\right)$ where

$$
\begin{aligned}
d_{2} a\left[s a_{1}|\ldots| s a_{k}\right]= & (-1)^{|a|} a a_{1}\left[s a_{2}|\ldots| s a_{k}\right] \\
& +\sum_{i=1}^{k-1}(-1)^{\varepsilon_{i}} a\left[s a_{1}|\ldots| s a_{i} a_{i+1}|\ldots| s a_{k}\right] \\
& -(-1)^{\left|s a_{k}\right| \varepsilon_{k-1}} a_{k} a\left[s a_{1}|\ldots| s a_{k-1}\right] .
\end{aligned}
$$

Here $\varepsilon_{i}=|a|+\left|s a_{1}\right|+\cdots+\left|s a_{i}\right|$.

Connes' boundary map $B$ is the map of degree +1

$$
B: A \otimes(s \bar{A})^{\otimes p} \rightarrow A \otimes(s \bar{A})^{\otimes p+1}
$$

defined by

$$
B\left(a_{o}\left[s a_{1}|\ldots| s a_{p}\right]\right)=\sum_{i=0}^{p}(-1)^{\left|s a_{0} \ldots s a_{i-1}\right|\left|s a_{i} \ldots s a_{p}\right|}\left[s a_{i}|\ldots| s a_{p}\left|s a_{0}\right| \ldots \mid s a_{p-1}\right] .
$$

Up to the isomorphism $s^{p}\left(A^{\otimes(p+1)}\right) \rightarrow A \otimes(s A)^{\otimes p}, s^{p}\left(a_{0}\left[a_{1}|\ldots| a_{p}\right]\right) \mapsto$ $(-1)^{p\left|a_{0}\right|+(p-1)\left|a_{1}\right|+\cdots+\left|a_{p-1}\right|} a_{0}\left[s a_{1}|\ldots| s a_{p}\right]$, our signs coincides with those of [29].

The Hochschild homology of $A$ (with coefficient in $A$ ) is the homology of the Hochschild chain complex:

$$
H H_{*}(A ; A):=H_{*}\left(\bigodot_{*}(A ; A)\right) .
$$

The Hochschild cohomology of $A$ (with coefficient in $A^{\vee}$ ) is the homology of the dual of the Hochschild chain complex:

$$
H H^{*}\left(A ; A^{\vee}\right):=H_{*}\left(\mathcal{C}_{*}(A ; A)^{\vee}\right) .
$$

Consider the dual of Connes' boundary map, $B^{\vee}(\varphi)=(-1)^{|\varphi|} \varphi \circ B$. On $H H^{*}\left(A ; A^{\vee}\right)$, $B^{\vee}$ defines an action of $H_{*}\left(S^{1}\right)$.

Example 12. Let $n \geq 2$. Let $\mathbb{k}$ be any commutative ring. Let $A:=H^{*}\left(S^{n}\right)=\Lambda x_{-n}$ be the exterior algebra on a generator of lower degree $-n$. Denote by $[s x]^{k}:=$ $1[s x|\ldots| s x]$ and $x[s x]^{k}:=x[s x|\ldots| s x]$ the elements of $\mathcal{C}_{*}(A ; A)$ where the term $s x$ appears $k$ times. These elements form a basis of $\mathcal{C}_{*}(A ; A)$. Denote by $[s x]^{k \vee}$, $x[s x]^{k \vee}, k \geq 0$, the dual basis. The differential $d^{\vee}$ on $\mathcal{C}_{*}(A ; A)^{\vee}$ is given by 
$d^{\vee}\left([s x]^{k \vee}\right)=0$ and $d^{\vee}\left(x[s x]^{k \vee}\right)= \pm\left(1-(-1)^{k(n+1)}\right)[s x]^{(k+1) \vee}$. The dual of Connes' boundary map $B^{\vee}$ is given by

$$
B^{\vee}\left([s x]^{k \vee}\right)= \begin{cases}(-1)^{n+1} k x[s x]^{(k-1) \vee} & \text { if }(k+1)(n+1) \text { is even, } \\ 0 & \text { if }(k+1)(n+1) \text { is odd, }\end{cases}
$$

and $B^{\vee}\left(x[s x]^{k \vee}\right)=0$. We remark that $[s x]^{k \vee}$ is of (lower) degree $k(n-1)$ and $x[s x]^{k \vee}$ of degree $n+k(n-1)$.

Theorem 13 ([17]). Let $X$ be a simply connected space such that $H_{*}(X ; \mathbb{k})$ is of finite type in each degree. Then there is a natural isomorphism of $H_{*}\left(S^{1}\right)$-modules between the homology of the free loop space on $X$ and the Hochschild cohomology of the algebra of singular cochain $S^{*}(X ; \mathbb{k})$ :

$$
H_{*}(L X) \cong H H^{*}\left(S^{*}(X ; \mathbb{k}) ; S^{*}(X ; \mathbb{k})^{\vee}\right) .
$$

In this paper, when we will apply this theorem, $H_{*}(X ; \mathbb{k})$ is assumed to be $\mathbb{k}$-free of finite type in each degree and $X$ will be always $\mathbb{k}$-formal: the algebra $S^{*}(X ; \mathbb{k})$ will be linked by quasi-isomorphisms of cochain algebras to $H_{*}(X ; \mathbb{k})$. Therefore

$$
H H^{*}\left(S^{*}(X ; \mathbb{k}) ; S^{*}(X ; \mathbb{k})^{\vee}\right) \cong H H^{*}\left(H^{*}(X ; \mathbb{k}) ; H^{*}(X ; \mathbb{k})^{\vee}\right) \text {. }
$$

Theorem 16. For $n>1$ odd, as a Batalin-Vilkovisky algebra,

$$
\begin{gathered}
\mathbb{H}_{*}\left(L S^{n} ; \mathbb{k}\right)=\mathbb{k}\left[u_{n-1}\right] \otimes \Lambda a_{-n}, \\
\Delta\left(u_{n-1}^{i} \otimes a_{-n}\right)=i\left(u_{n-1}^{i-1} \otimes 1\right), \\
\Delta\left(u_{n-1}^{i} \otimes 1\right)=0 .
\end{gathered}
$$

Proof. For the algebra structure, Cohen, Jones and Yan [8] proved that $\mathbb{H}_{*}\left(L S^{n} ; \mathbb{Z}\right)=$ $\mathbb{k}\left[u_{n-1}\right] \otimes \Lambda a_{-n}$ when $\mathbb{k}=\mathbb{Z}$. Their proof works over any $\mathbb{k}$ (alternatively, using Proposition 9 , we could assume that $\mathbb{k}=\mathbb{Z}$ ). Computing Connes' boundary map on $H H^{*}\left(H^{*}\left(S^{n}\right) ; H_{*}\left(S^{n}\right)\right)$ (Example 12$)$, we see that $\Delta$ on $\mathbb{H}_{*}\left(L S^{n} ; \mathbb{k}\right)$ is null in even degree and in degree $-n$, and is an isomorphism in degree -1 . Therefore $\Delta\left(u_{n-1}^{i} \otimes 1\right)=0, \Delta\left(1 \otimes a_{-n}\right)=0$ and $\Delta\left(u_{n-1} \otimes a_{-n}\right)=\alpha 1$ where $\alpha$ is invertible in $\mathbb{k}$. Replacing $a_{-n}$ by $\frac{1}{\alpha} a_{-n}$ or $u_{n-1}$ by $\frac{1}{\alpha} u_{n-1}$, we can assume up to isomorphisms that $\Delta\left(u_{n-1} \otimes a_{-n}\right)=1$. Therefore $\left\{u_{n-1}, a_{-n}\right\}=1$. Using the Poisson relation (7), $\left\{u_{n-1}^{i}, a_{-n}\right\}=i u_{n-1}^{i-1}$. Therefore $\Delta\left(u_{n-1}^{i} \otimes a_{-n}\right)=i\left(u_{n-1}^{i-1} \otimes 1\right)$. 
Theorem 17. For $n \geq 2$ even, there exists a constant $\varepsilon_{0} \in \mathbb{F}_{2}$ such that as a BatalinVilkovisky algebra,

$$
\begin{aligned}
\mathbb{H}_{*}\left(L S^{n} ; \mathbb{Z}\right) & =\Lambda b \otimes \frac{\mathbb{Z}[a, v]}{\left(a^{2}, a b, 2 a v\right)} \\
& =\bigoplus_{k=0}^{+\infty} \mathbb{Z} v_{2(n-1)}^{k} \oplus \bigoplus_{k=0}^{+\infty} \mathbb{Z} b_{-1} v^{k} \oplus \mathbb{Z} a_{-n} \oplus \bigoplus_{k=1}^{+\infty} \frac{\mathbb{Z}}{2 \mathbb{Z}} a v^{k},
\end{aligned}
$$

for all $k \geq 0, \Delta\left(v^{k}\right)=0, \Delta\left(a v^{k}\right)=0$ and

$$
\Delta\left(b v^{k}\right)= \begin{cases}(2 k+1) v^{k}+\varepsilon_{0} a v^{k+1} & \text { if } n=2 \\ (2 k+1) v^{k} & \text { if } n \geq 4\end{cases}
$$

Proof. For the algebra structure, Cohen, Jones and Yan [8] proved the equality. Computing Connes' boundary map on $H H^{*}\left(H^{*}\left(S^{n}\right) ; H_{*}\left(S^{n}\right)\right)$ (Example 12), we see that $\Delta$ on $\mathbb{H}_{*}\left(L S^{n} ; \mathbb{k}\right)$ is null in even degree and is injective in odd degree.

Case $n \neq 2$. This case is simple, since all the generators of $\mathbb{H}_{*}\left(L S^{n}\right), v^{k}, b v^{k}$ and $a v^{k}, k \geq 0$, have different degrees. Using Example 12, we also see that for all $k \geq 0$,

$$
\Delta: \mathbb{H}_{-1+2 k(n-1)}=\mathbb{Z} b_{-1} v^{k} \hookrightarrow \mathbb{H}_{2 k(n-1)}=\mathbb{Z} v^{k}
$$

has cokernel isomorphic to $\frac{\mathbb{Z}}{(2 k+1) \mathbb{Z}}$. Therefore $\Delta\left(b v^{k}\right)= \pm(2 k+1) v^{k}$. By replacing $b_{-1}$ by $-b_{-1}$, we can assume up to isomorphims that $\Delta(b)=1$. Let $k \geq 1$. Let $\alpha_{k} \in\{-2 k-1,2 k+1\}$ such that $\Delta\left(b v^{k}\right)=\alpha_{k} v^{k}$. Using formula (6), we obtain that $\Delta\left(b v^{k} v^{k}\right)=\left(2 \alpha_{k}-1\right) v^{2 k}$. We know that $\Delta\left(b v^{2 k}\right)= \pm(4 k+1) v^{2 k}$. Therefore $\alpha_{k}$ must be equal to $2 k+1$.

Case $n=2$. This case is complicated, since for $k \geq 0, v^{k}$ and $a v^{k+1}$ have the same degree. Using Example 12, we also see that

$$
\Delta: \mathbb{H}_{-1+2 k}=\mathbb{Z} b_{-1} v^{k} \hookrightarrow \mathbb{H}_{2 k}=\mathbb{Z} v^{k} \oplus \frac{\mathbb{Z}}{2 \mathbb{Z}} a v^{k+1}
$$

has cokernel, denoted $\operatorname{Coker} \Delta$, isomorphic to $\frac{\mathbb{Z}}{(2 k+1) \mathbb{Z}} \oplus \frac{\mathbb{Z}}{2 \mathbb{Z}}$. There exists unique $\alpha_{k} \in \mathbb{Z}^{*}$ and $\varepsilon_{k} \in \frac{\mathbb{Z}}{2 \mathbb{Z}}$ such that $\Delta\left(b v^{k}\right)=\alpha_{k} v^{k}+\varepsilon_{k} a v^{k+1}$. The injective map $\Delta$ 
fits into the commutative diagram of short exact sequences (Noether's Lemma)

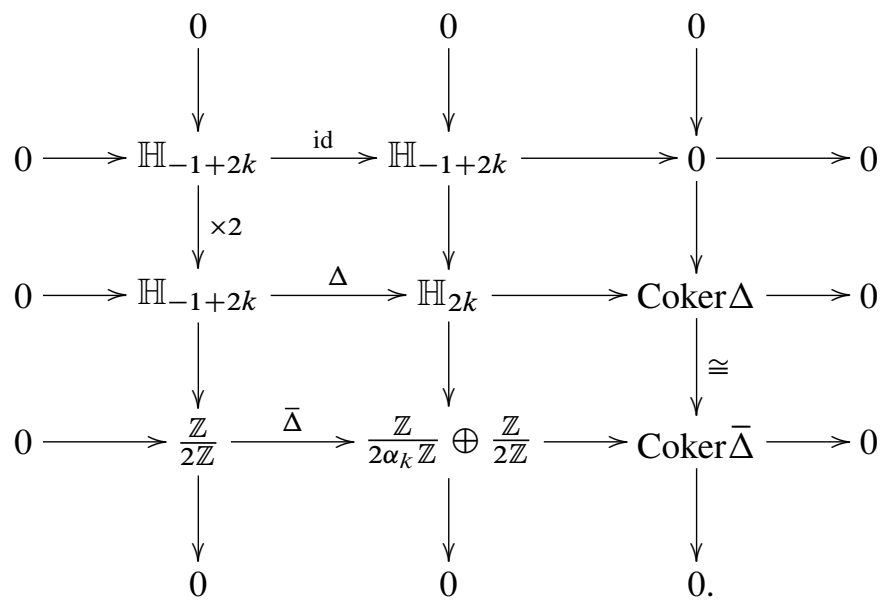

The cokernel of $\bar{\Delta}$, denoted Coker $\bar{\Delta}$ is of cardinal $2\left|\alpha_{k}\right|$. So $\left|\alpha_{k}\right|=2 k+1$. Therefore $\Delta\left(b v^{k}\right)= \pm(2 k+1) v^{k}+\varepsilon_{k} a v^{k+1}$.

By replacing $b_{-1}$ by $-b_{-1}$, we can assume up to isomorphims that $\Delta(b)=$ $1+\varepsilon_{0} a v$. Using formula (6), we obtain that

$$
\Delta\left(b v^{k} v^{l}\right)=\left(\alpha_{k}+\alpha_{l}-1\right) v^{k+l}+\left(\varepsilon_{k}+\varepsilon_{l}-\varepsilon_{0}\right) a v^{k+l+1} .
$$

Therefore

$$
\Delta\left(b v^{k} v^{k}\right)=\left(2 \alpha_{k}-1\right) v^{2 k}+\varepsilon_{0} a v^{2 k+1}= \pm(4 k+1) v^{2 k}+\varepsilon_{2 k} a v^{2 k+1} .
$$

So $\alpha_{k}=2 k+1, \varepsilon_{2 k}=\varepsilon_{0}$ and $\varepsilon_{2 k+1}=\varepsilon_{2 k}+\varepsilon_{1}-\varepsilon_{0}=\varepsilon_{1}$.

The map $\Theta: \mathbb{H}_{*}\left(L S^{2}\right) \rightarrow \mathbb{H}_{*}\left(L S^{2}\right)$ given by $\Theta\left(b_{-1} v^{k}\right)=b_{-1} v^{k}, \Theta\left(v^{k}\right)=$ $v^{k}+k a v^{k+1}, \Theta\left(a v^{k}\right)=a v^{k}, k \geq 0$ is an involutive isomorphism of algebras. Therefore, by replacing $v$ by $v+a v^{2}$, we can assume that $\varepsilon_{1}=\varepsilon_{0}$. So we have proved

$$
\Delta\left(b v^{k}\right)=(2 k+1) v^{k}+\varepsilon_{0} a v^{k+1}, \quad k \geq 0 .
$$

These two cases $\varepsilon_{0}=0$ and $\varepsilon_{0}=1$ correspond to two non-isomorphic BatalinVilkovisky algebras whose underlying Gerstenhaber algebras are the same. Therefore even if we have not yet computed the Batalin-Vilkovisky algebra $\mathbb{H}_{*}\left(L S^{2} ; \mathbb{Z}\right)$, we have computed its underlying Gerstenhaber algebra. Using the definition of the bracket, straightforward computations give the following corollary.

Corollary 18. For $n \geq 2$ even, as Gerstenhaber algebra

$$
\mathbb{H}_{*}\left(L S^{n} ; \mathbb{Z}\right)=\Lambda b_{-1} \otimes \frac{\mathbb{Z}\left[a_{-n}, v_{2(n-1)}\right]}{\left(a^{2}, a b, 2 a v\right)}
$$


with $\left\{v^{k}, v^{l}\right\}=0,\left\{b v^{k}, v^{l}\right\}=-2 l v^{k+l},\left\{b v^{k}, b v^{l}\right\}=2(k-l) b v^{k+l},\left\{a, v^{l}\right\}=0$, $\left\{a v^{k}, b v^{l}\right\}=-(2 l+1) a v^{k+l}$ and $\left\{a v^{k}, a v^{l}\right\}=0$ for all $k, l \geq 0$.

\section{When Hochschild cohomology is a Batalin-Vilkovisky algebra}

In this section, we recall the structure of Gerstenhaber algebra on the Hochschild cohomology of an algebra whose degrees are bounded. We recall from [26], [22], [27], [19] the Batalin-Vilkovisky algebra on the Hochschild cohomology of the cohomology $H^{*}(M)$ of a closed oriented manifold $M$. We compute this Batalin-Vilkovisky algebra $H H^{*}\left(H^{*}(M) ; H^{*}(M)\right)$ when $M$ is a sphere.

Throughout in this section we will work over the prime field $\mathbb{F}_{2}$. Let $A$ be an augmented graded algebra such that the augmentation ideal $\bar{A}$ is concentrated in degree $\leq-2$ and bounded below (or concentrated in degree $\geq 0$ and bounded above). Then the (normalized) Hochschild cochain complex, denoted $\mathcal{C}^{*}(A, A)$, is the complex

$$
\operatorname{Hom}(T s \bar{A}, A) \cong \bigoplus_{p \geq 0} \operatorname{Hom}\left((s \bar{A})^{\otimes p}, A\right)
$$

with a differential $d_{2}$. For an element $f$ in $\operatorname{Hom}\left((s \bar{A})^{\otimes p}, A\right)$, the differential $d_{2} f$ in $\operatorname{Hom}\left((s \bar{A})^{\otimes p+1}, A\right)$ is given by

$$
\begin{aligned}
& \left(d_{2} f\right)\left(\left[s a_{1}|\ldots| s a_{p+1}\right]\right):=a_{1} f\left(\left[s a_{2}|\ldots| s a_{p+1}\right]\right) \\
& \quad+\sum_{i=1}^{p} f\left(\left[s a_{1}|\ldots| s\left(a_{i} a_{i+1}\right)|\ldots| s a_{p+1}\right]\right)+f\left(\left[s a_{1}|\ldots| s a_{p}\right]\right) a_{p} .
\end{aligned}
$$

The Hochschild cohomology of $A$ with coefficient in $A$ is the homology of the Hochschild cochain complex:

$$
H H^{*}(A ; A):=H_{*}\left(\mathcal{C}^{*}(A ; A)\right) .
$$

We remark that $H H^{*}(A ; A)$ is bigraded. Our degree is sometimes called the total degree: sum of the external degree and the internal degree. The Hochschild cochain complex $\mathcal{C}^{*}(A, A)$ is a differential graded algebra. For $f \in \operatorname{Hom}\left((s \bar{A})^{\otimes p}, A\right)$ and $g \in \operatorname{Hom}\left((s \bar{A})^{\otimes q}, A\right)$, the (cup) product of $f$ and $g, f \cup g \in \operatorname{Hom}\left((s \bar{A})^{\otimes p+q}, A\right)$ is defined by

$$
(f \cup g)\left(\left[s a_{1}|\ldots| s a_{p+q}\right]\right):=f\left(\left[s a_{1}|\ldots| s a_{p}\right]\right) g\left(\left[s a_{p+1}|\ldots| s a_{p+q}\right]\right) .
$$

The Hochschild cochain complex $\varrho^{*}(A, A)$ has also a Lie bracket of (lower) degree +1 .

$$
\begin{aligned}
(f \bar{\circ} g) & \left(\left[s a_{1}|\ldots| s a_{p+q-1}\right]\right) \\
& :=\sum_{i=1}^{p} f\left(\left[s a_{1}|\ldots| s a_{i-1}\left|s g\left(\left[s a_{i}|\ldots| s a_{i+q-1}\right]\right)\right| s a_{i+q}|\ldots| s a_{p+q-1}\right]\right) .
\end{aligned}
$$


$\{f, g\}=f \bar{\circ} g-g \bar{\circ} f$. Our formulas are the same as in the non-graded case [13]. We remark that if $A$ is not assumed to be bounded, the formulas are more complicated. Gerstenhaber has shown that $H H^{*}(A ; A)$ equipped with the cup product and the Lie bracket is a Gerstenhaber algebra.

Let $M$ be a closed $d$-dimensional smooth manifold. Poincaré duality induces an isomorphism of $H^{*}\left(M ; \mathbb{F}_{2}\right)$-modules of (lower) degree $d$ :

$$
\Theta: H^{*}\left(M ; \mathbb{F}_{2}\right) \stackrel{\cap[M]}{\longrightarrow} H_{*}\left(M ; \mathbb{F}_{2}\right) \cong H^{*}\left(M ; \mathbb{F}_{2}\right)^{\vee} .
$$

More generally, let $A$ be a graded algebra equipped with an isomorphism of $A$ bimodules of degree $d, \Theta: A \stackrel{\cong}{\longrightarrow} A^{\vee}$. Then we have the isomorphism

$$
H H^{*}(A, \Theta): H H^{*}(A, A) \stackrel{\cong}{\longrightarrow} H H^{*}\left(A, A^{\vee}\right) .
$$

Therefore on $H H^{*}(A, A)$, we have both a Gerstenhaber algebra structure and an operator $\Delta$ given by the dual of Connes' boundary map $B$. Motivated by the BatalinVilkovisky algebra structure of Chas-Sullivan on $\mathbb{H}_{*}(L M)$, Thomas Tradler [26] proved that $H H^{*}(A, A)$ is a Batalin-Vilkovisky algebra. See [22, Theorem 1.6] for an explicit proof. In [19] or [27, Corollary 3.4] or [9, Section 1.4] or [20, Theorem B] or [21, Section 11.6], this Batalin-Vilkovisky algebra structure on $H H^{*}(A, A)$ extends to a structure of algebra on the Hochschild cochain complex $\ell^{*}(A, A)$ over various operads or PROPs: the so-called cyclic Deligne conjecture. Let us compute this Batalin-Vilkovisky algebra structure when $M$ is a sphere.

Proposition 20 ([30] and [31, Corollary 4.2]). Let $d \geq 2$. As Batalin-Vilkovisky algebra, for the Hochschild cohomology of $H^{*}\left(S^{d} ; \mathbb{F}_{2}\right)=\Lambda x_{-d}$, we have

$$
H H^{*}\left(H^{*}\left(S^{d} ; \mathbb{F}_{2}\right) ; H^{*}\left(S^{d} ; \mathbb{F}_{2}\right)\right) \cong \Lambda g_{-d} \otimes \mathbb{F}_{2}\left[f_{d-1}\right]
$$

with $\Delta\left(g_{-d} \otimes f_{d-1}^{k}\right)=k\left(1 \otimes f_{d-1}^{k-1}\right)$ and $\Delta\left(1 \otimes f_{d-1}^{k}\right)=0, k \geq 0$. In particular, the underlying Gerstenhaber algebra is given by $\left\{f^{k}, f^{l}\right\}=0,\left\{g f^{k}, f^{l}\right\}=l f^{k+l-1}$ and $\left\{g f^{k}, g f^{l}\right\}=(k-l) g f^{k+l-1}$ for $k, l \geq 0$.

Proof. Denote by $A:=H^{*}\left(S^{d} ; \mathbb{F}_{2}\right)$. The differential on $\complement^{*}(A ; A)$ is null. Let $f \in \operatorname{Hom}(s \bar{A}, A) \subset \mathcal{C}^{*}(A ; A)$ such that $f([s x])=1$. Let $g \in \operatorname{Hom}\left(\mathbb{F}_{2}, A\right)=$ $\operatorname{Hom}\left((s \bar{A})^{\otimes 0}, A\right) \subset \mathcal{C}^{*}(A ; A)$ such that $g([])=x$. The $k$-th power of $f$ is the map $f^{k} \in \operatorname{Hom}\left((s \bar{A})^{\otimes k}, A\right)$ such that $f^{k}([s x|\ldots| s x])=1$. The cup product $g \cup f^{k} \in \operatorname{Hom}\left((s \bar{A})^{\otimes k}, A\right)$ sends $[s x|\ldots| s x]$ to $x$. So we have proved that $\mathcal{C}^{*}(A ; A)$ is isomorphic to the tensor product of graded algebras $\Lambda g_{-d} \otimes \mathbb{F}_{2}\left[f_{d-1}\right]$.

The unit 1 and $x_{-d}$ form a linear basis of $H^{*}\left(S^{d}\right)$. Denote by $1^{\vee}$ and $x^{\vee}$ the dual basis of $A^{\vee}=H^{*}\left(S^{d}\right)^{\vee}$. Poincaré duality induces the isomorphism $\Theta: H^{*}\left(S^{d}\right) \stackrel{\cong}{\longrightarrow} H^{*}\left(S^{d}\right)^{\vee}, 1 \mapsto x^{\vee}$ and $x \mapsto 1^{\vee}$. The two families of elements of 
the form $1[s x|\ldots| s x]$ and of the form $x[s x|\ldots| s x]$ form a basis of $\mathcal{C}_{*}(A ; A)$. Denote by $1[s x|\ldots| s x]^{\vee}$ and $x[s x|\ldots| s x]^{\vee}$ the dual basis in $\mathcal{C}_{*}(A ; A)^{\vee}$. The isomor-

phism $\Theta$ induces an isomorphism of complexes of degree $d, \widehat{\Theta}: \mathcal{C}^{*}(A ; A) \stackrel{\mathcal{C}^{*}(A ; \Theta)}{\cong}$ $\complement^{*}\left(A ; A^{\vee}\right) \stackrel{\cong}{\longrightarrow} \bigodot_{*}(A ; A)^{\vee}$. Explicitly [22, Section 4] this isomorphism sends $f \in \operatorname{Hom}\left((s \bar{A})^{\otimes p}, A\right)$ to the linear map $\widehat{\Theta}(f) \in\left(A \otimes(s \bar{A})^{\otimes p}\right)^{\vee} \subset \mathcal{C}_{*}(A ; A)^{\vee}$ defined by

$$
\widehat{\Theta}(f)\left(a_{0}\left[s a_{1}|\ldots| s a_{p}\right]\right)=\left((\Theta \circ f)\left[s a_{1}|\ldots| s a_{p}\right]\right)\left(a_{0}\right) .
$$

Here with $A=\Lambda x, \widehat{\Theta}\left(f^{k}\right)=x[s x|\ldots| s x]^{\vee}$ and $\widehat{\Theta}\left(g \cup f^{k}\right)=1[s x|\ldots| s x]^{\vee}$. Computing Connes' boundary map $B^{\vee}$ on $\ell_{*}(A ; A)^{\vee}$ (Example 12) and using that $\widehat{\Theta} \circ \Delta=B^{\vee} \circ \widehat{\Theta}$ by definition of $\Delta$, we obtain the desired formula for $\Delta$.

\section{The Gerstenhaber algebra $\mathbb{H}_{*}\left(L S^{2} ; \mathbb{F}_{2}\right)$}

Using the same Hochschild homology technique as in Section 4, we compute, up to an indeterminacy, the Batalin-Vilkovisky algebra $\mathbb{H}_{*}\left(L S^{2} ; \mathbb{F}_{2}\right)$. Nevertheless, this will give the complete description of the underlying Gerstenhaber algebra on $\mathbb{H}_{*}\left(L S^{2} ; \mathbb{F}_{2}\right)$.

Lemma 21. There exists a constant $\varepsilon \in\{0,1\}$ such that as a Batalin-Vilkovisky algebra, the homology of the free space loop on the sphere $S^{2}$ is

$$
\begin{gathered}
\mathbb{H}_{*}\left(L S^{2} ; \mathbb{F}_{2}\right)=\Lambda a_{-2} \otimes \mathbb{F}_{2}\left[u_{1}\right], \\
\Delta\left(a_{-2} \otimes u_{1}^{k}\right)=k\left(1 \otimes u_{1}^{k-1}+\varepsilon a_{-2} \otimes u_{1}^{k+1}\right) \text { and } \Delta\left(1 \otimes u_{1}^{k}\right)=0, \quad k \geq 0 .
\end{gathered}
$$

Proof. In [8], Cohen, Jones and Yan proved that the Serre spectral sequence for the free loop fibration $\Omega M \stackrel{j}{\hookrightarrow} L M \stackrel{\mathrm{ev}}{\longrightarrow} M$ is a spectral sequence of algebras converging toward the algebra $\mathbb{H}_{*}(L M)$. Using Hochschild homology, we see that there is an isomorphism of vector spaces $\mathbb{H}_{*}\left(L S^{2} ; \mathbb{F}_{2}\right) \cong \mathbb{H}_{*}\left(S^{2} ; \mathbb{F}_{2}\right) \otimes H_{*}\left(\Omega S^{2} ; \mathbb{F}_{2}\right)$. Therefore the Serre spectral sequence collapses. Since there is no extension problem, we have the isomorphism of algebras

$$
\mathbb{H}_{*}\left(L S^{2} ; \mathbb{F}_{2}\right) \cong \mathbb{H}_{*}\left(S^{2} ; \mathbb{F}_{2}\right) \otimes H_{*}\left(\Omega S^{2} ; \mathbb{F}_{2}\right)=\Lambda\left(a_{-2}\right) \otimes \mathbb{F}_{2}\left[u_{1}\right]
$$

Computing Connes' boundary map on $H H^{*}\left(H^{*}\left(S^{2} ; \mathbb{F}_{2}\right) ; H_{*}\left(S^{2} ; \mathbb{F}_{2}\right)\right.$ ) (see Example 12), we see that $\Delta$ on $\mathbb{H}_{*}\left(L S^{2} ; \mathbb{F}_{2}\right)$ is null in even degree and that

$$
\Delta: \mathbb{H}_{2 k-1} \rightarrow \mathbb{H}_{2 k}
$$


is a linear map of rank $1, k \geq 0$. In particular $\Delta$ is injective in degree -1 .

Applying Lemma 11 to the identity map id: $S^{2} \rightarrow S^{2}$, we see that the composite

$$
H_{1}\left(\Omega S^{2} ; \mathbb{F}_{2}\right) \stackrel{H_{1}\left(j ; \mathbb{F}_{2}\right)}{\longrightarrow} H_{1}\left(L S^{2} ; \mathbb{F}_{2}\right) \stackrel{\Delta}{\longrightarrow} H_{2}\left(L S^{2} ; \mathbb{F}_{2}\right) \stackrel{H_{2}\left(\mathrm{ev} ; \mathbb{F}_{2}\right)}{\longrightarrow} H_{2}\left(S^{2} ; \mathbb{F}_{2}\right)
$$

is non-zero. Since $\mathbb{H}_{*}(\mathrm{ev})$ is a morphism of algebras, $\mathbb{H}_{0}(e v)\left(a_{-2} u_{1}^{2}\right)=0$. And so $\Delta\left(a_{-2} u_{1}\right)=1+\varepsilon a_{-2} u_{1}^{2}$ with $\varepsilon \in \mathbb{F}_{2}$.

We remark that when $b=c$, formula (6) takes the simple form

$$
\Delta\left(a b^{2}\right)=\Delta(a) b^{2}+a \Delta\left(b^{2}\right) .
$$

Using this formula, we obtain that

$$
\Delta\left(a_{-2} u_{1}^{2 k+1}\right)=\Delta\left(\left(a_{-2} u_{1}\right)\left(u_{1}^{k}\right)^{2}\right)=u_{1}^{2 k}+\varepsilon a_{-2} u_{1}^{2 k+2}, \quad k \geq 0 .
$$

Since $\Delta: \mathbb{H}_{1}=\mathbb{F}_{2} a_{-2} u_{1}^{3} \oplus \mathbb{F}_{2} u_{1} \rightarrow \mathbb{H}_{2}$ is of rank 1 and $\Delta\left(a_{-2} u_{1}^{3}\right) \neq 0, \Delta\left(u_{1}\right)=$ $\lambda \Delta\left(a_{-2} u_{1}^{3}\right)$ with $\lambda=0$ or $\lambda=1$. Using again formula (22), we have that

$$
\Delta\left(u_{1}^{2 k+1}\right)=\Delta\left(u_{1}\left(u_{1}^{k}\right)^{2}\right)=\lambda \Delta\left(a_{-2} u_{1}^{3}\right) u_{1}^{2 k}=\lambda \Delta\left(a_{-2} u_{1}^{2 k+3}\right), \quad k \geq 0 .
$$

So finally

$$
\Delta\left(a_{-2} u_{1}^{k}\right)=k u_{1}^{k-1}+\varepsilon k a_{-2} u_{1}^{k+1} \text { and } \Delta\left(u_{1}^{k}\right)=\lambda \Delta\left(a_{-2} u_{1}^{k+2}\right), \quad k \geq 0 .
$$

The cases $\lambda=0$ and $\lambda=1$ correspond to isomorphic Batalin-Vilkovisky algebras: Let $\Theta: \mathbb{H}_{*}\left(L S^{2} ; \mathbb{F}_{2}\right) \rightarrow \mathbb{H}_{*}\left(L S^{2} ; \mathbb{F}_{2}\right)$ be an automorphism of algebras which is not the identity. Since $\Theta\left(a_{-2}\right) \neq 0, \Theta\left(a_{-2}\right)=a_{-2}$. Since $\Theta\left(a_{-2}\right)$ and $\Theta\left(u_{1}\right)$ must generate the algebra $\Lambda a_{-2} \otimes \mathbb{F}_{2}\left[u_{1}\right], \Theta\left(u_{1}\right) \neq a_{-2} u_{1}^{3}$. Since $\Theta\left(u_{1}\right) \neq u_{1}$, $\Theta\left(u_{1}\right)=u_{1}+a_{-2} u_{1}^{3}$. Therefore there is an unique automorphism of algebras $\Theta: \mathbb{H}_{*}\left(L S^{2} ; \mathbb{F}_{2}\right) \rightarrow \mathbb{H}_{*}\left(L S^{2} ; \mathbb{F}_{2}\right)$ which is not the identity. Explicitly, $\Theta$ is given by $\Theta\left(u_{1}^{k}\right)=u_{1}^{k}+k a_{-2} u_{1}^{k+2}, \Theta\left(a_{-2} u_{1}^{k}\right)=a_{-2} u_{1}^{k}, k \geq 0$. One can check that $\Theta$ is an involutive isomorphism of Batalin-Vilkovisky algebras who transforms the cases $\lambda=0$ into the cases $\lambda=1$ without changing $\varepsilon$. Therefore, by replacing $u_{1}$ by $u_{1}+a_{-2} u_{1}^{3}$, we can assume that $\lambda=0$.

Consider the Batalin-Vilkovisky algebras $\Lambda a_{-2} \otimes \mathbb{F}_{2}\left[u_{1}\right]$ with $\Delta\left(a_{-2} \otimes u_{1}^{k}\right)=$ $k\left(1 \otimes u_{1}^{k-1}+\varepsilon a_{-2} \otimes u_{1}^{k+1}\right), \Delta\left(1 \otimes u_{1}^{k}\right)=\lambda \Delta\left(a_{-2} u_{1}^{k+2}\right), k \geq 0$, given by the different values of $\varepsilon, \lambda \in\{0,1\}$. These four Batalin-Vilkovisky algebras have only two underlying Gerstenhaber algebras given by $\left\{u_{1}^{k}, u_{1}^{l}\right\}=0,\left\{a_{-2} u_{1}^{k}, u_{1}^{l}\right\}=$ $l u^{k+l-1}+l(\varepsilon-\lambda) a_{-2} u^{k+l+1}$ and $\left\{a_{-2} u_{1}^{k}, a_{-2} u_{1}^{l}\right\}=(k-l) a_{-2} u^{k+l-1}$ for $k, l \geq 0$. Via the above isomorphism $\Theta$, these two Gerstenhaber algebras are isomorphic.

Corollary 23. The free loop space modulo 2 homology $\mathbb{H}_{*}\left(L S^{2} ; \mathbb{F}_{2}\right)$ is isomorphic as Gerstenhaber algebra to the Hochschild cohomology of $H^{*}\left(S^{2} ; \mathbb{F}_{2}\right)$,

$$
H H^{*}\left(H^{*}\left(S^{2} ; \mathbb{F}_{2}\right) ; H^{*}\left(S^{2} ; \mathbb{F}_{2}\right)\right) \text {. }
$$




\section{The Batalin-Vilkovisky algebra $\mathbb{H}_{*}\left(L S^{2}\right)$}

In this section, we complete the calculations of the Batalin-Vilkovisky algebras $\mathbb{H}_{*}\left(L S^{2} ; \mathbb{F}_{2}\right)$ and $\mathbb{H}_{*}\left(L S^{2} ; \mathbb{Z}\right)$ started respectively in Sections 6 and 4 , using a purely homotopic method.

Theorem 24. As a Batalin-Vilkovisky algebra, the homology of the free loop space on the sphere $S^{2}$ with mod 2 coefficients is

$$
\begin{gathered}
\mathbb{H}_{*}\left(L S^{2} ; \mathbb{F}_{2}\right)=\Lambda a_{-2} \otimes \mathbb{F}_{2}\left[u_{1}\right], \\
\Delta\left(a_{-2} \otimes u_{1}^{k}\right)=k\left(1 \otimes u_{1}^{k-1}+a_{-2} \otimes u_{1}^{k+1}\right) \text { and } \Delta\left(1 \otimes u_{1}^{k}\right)=0, \quad k \geq 0 .
\end{gathered}
$$

Theorem 25. With integer coefficients, as a Batalin-Vilkovisky algebra,

$$
\begin{aligned}
\mathbb{H}_{*}\left(L S^{2} ; \mathbb{Z}\right) & =\Lambda b \otimes \frac{\mathbb{Z}[a, v]}{\left(a^{2}, a b, 2 a v\right)} \\
= & \bigoplus_{k=0}^{+\infty} \mathbb{Z} v_{2}^{k} \oplus \bigoplus_{k=0}^{+\infty} \mathbb{Z} b_{-1} v^{k} \oplus \mathbb{Z} a_{-2} \oplus \bigoplus_{k=1}^{+\infty} \frac{\mathbb{Z}}{2 \mathbb{Z}} a v^{k}
\end{aligned}
$$

for all $k \geq 0, \Delta\left(v^{k}\right)=0, \Delta\left(a v^{k}\right)=0$ and $\Delta\left(b v^{k}\right)=(2 k+1) v^{k}+a v^{k+1}$.

Denote by $s: X \hookrightarrow L X$ the trivial section of the evaluation map ev: $L X \rightarrow X$.

Lemma 26. The image of $\Delta: H_{1}\left(L S^{2} ; \mathbb{F}_{2}\right) \rightarrow H_{2}\left(L S^{2} ; \mathbb{F}_{2}\right)$ is not contained in the image of $H_{2}\left(s ; \mathbb{F}_{2}\right): H_{2}\left(S^{2} ; \mathbb{F}_{2}\right) \hookrightarrow H_{2}\left(L S^{2} ; \mathbb{F}_{2}\right)$.

Lemma 27. The image of $\Delta: H_{1}\left(L S^{2} ; \mathbb{Z}\right) \rightarrow H_{2}\left(L S^{2} ; \mathbb{Z}\right)$ is not contained in the image of $\mathrm{H}_{2}(s ; \mathbb{Z}): H_{2}\left(S^{2} ; \mathbb{Z}\right) \hookrightarrow H_{2}\left(L S^{2} ; \mathbb{Z}\right)$.

Proof of Lemma 27 assuming Lemma 26. Consider the commutative diagram

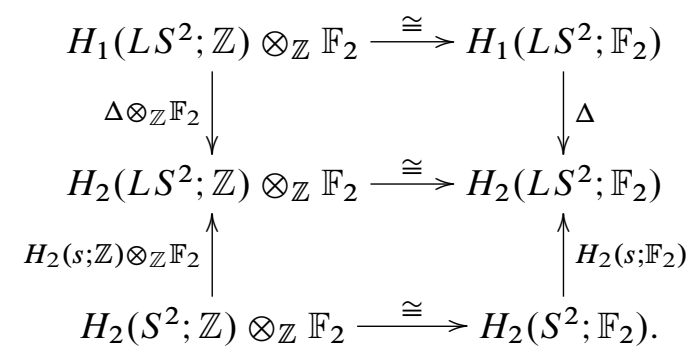

Since $H_{1}\left(L S^{2} ; \mathbb{Z}\right) \cong H_{0}\left(L S^{2} ; \mathbb{Z}\right) \cong \mathbb{Z}$, the horizontal arrows are isomorphisms by the universal coefficient theorem. The top rectangle commutes according to Lemma 4. 
Suppose that the image of $\Delta: H_{1}\left(L S^{2} ; \mathbb{Z}\right) \rightarrow H_{2}\left(L S^{2} ; \mathbb{Z}\right)$ is included in the image of $H_{2}(s ; \mathbb{Z})$. Then the image of $\Delta \otimes_{\mathbb{Z}} \mathbb{F}_{2}$ is included in the image of $H_{2}(s ; \mathbb{Z}) \otimes_{\mathbb{Z}} \mathbb{F}_{2}$. Using the above diagram, the image of $\Delta: H_{1}\left(L S^{2} ; \mathbb{F}_{2}\right) \rightarrow$ $H_{2}\left(L S^{2} ; \mathbb{F}_{2}\right)$ is included in the image of $H_{2}\left(s ; \mathbb{F}_{2}\right)$. This contradicts Lemma 26.

Proof of Theorem 24 assuming Lemma 26. It suffices to show that the constant $\varepsilon$ in Lemma 21 is not zero. Suppose that $\varepsilon=0$. Then by Lemma $21, \Delta\left(a_{-2} \otimes u_{1}\right)=1$.

It is well known that $\mathbb{H}_{*}(s): \mathbb{H}_{*}(M) \rightarrow \mathbb{H}_{*}(L M)$ is a morphism of algebras. In particular, let $\left[S^{2}\right]$ be the fundamental class of $S^{2}, H_{2}(s)\left(\left[S^{2}\right]\right)$ is the unit of $\mathbb{H}_{*}\left(L S^{2}\right)$. So $\Delta\left(a_{-2} \otimes u_{1}\right)=H_{2}(s)\left(\left[S^{2}\right]\right)$. This contradicts Lemma 26.

The proof of Theorem 25 assuming Lemma 27 is the same. To complete the computation of this Batalin-Vilkovisky algebra on the homology of the free loop space of a manifold, we will relate it to another structure of a Batalin-Vilkovisky algebra that arises in algebraic topology: the homology of the double loop space.

Let $X$ be a pointed topological space. The circle $S^{1}$ acts on the sphere $S^{2}$ by "rotating the earth". Hence the circle also acts on $\Omega^{2} X=\operatorname{map}\left(\left(S^{2}\right.\right.$, North pole $\left.),(X, *)\right)$. So we have an induced operator $\Delta: H_{*}\left(\Omega^{2} X\right) \rightarrow H_{*+1}\left(\Omega^{2} X\right)$. With Theorem 32 and the following proposition, we will able to prove Lemma 26.

Proposition 28. Let $X$ be a pointed topological space. There is a natural morphism $r: L \Omega X \rightarrow \operatorname{map}_{*}\left(S^{2}, X\right)$ of $S^{1}$-spaces between the free loop space on the pointed loops of $X$ and the double pointed loop space of $X$ such that:

- If we identify $S^{2}$ and $S^{1} \wedge S^{1}, r$ is a retract up to homotopy of the inclusion $j: \Omega(\Omega X) \hookrightarrow L(\Omega X)$.

- The composite $r \circ s: \Omega X \hookrightarrow L(\Omega X) \rightarrow \operatorname{map}_{*}\left(S^{2}, X\right)$ is homotopically trivial.

Proof. Let $\sigma: S^{2} \rightarrow \frac{S^{1} \times S^{1}}{S^{1} \times *}=S_{+}^{1} \wedge S^{1}$ be the quotient map that identifies the North pole and the South pole on the earth $S^{2}$. The circle $S^{1}$ acts without moving the based point on $S_{+}^{1} \wedge S^{1}$ by multiplication on the first factor. On the torus $S^{1} \times S^{1}$, the circle can act by multiplication on both factors. But when you pinch a circle to a point in the torus, the circle can act only on one factor. If we make a picture, we easily see that $\sigma: S^{2} \rightarrow S_{+}^{1} \wedge S^{1}$ is compatible with the actions of $S^{1}$. Therefore $r:=\operatorname{map}_{*}(\sigma, X): L \Omega X \rightarrow \operatorname{map}_{*}\left(S^{2}, X\right)$ is a morphism of $S^{1}$-spaces.

- Let $\pi: S_{+}^{1} \wedge S^{1} \rightarrow S^{1} \wedge S^{1}=\frac{S_{+}^{1} \wedge S^{1}}{* \times S^{1}}$ be the quotient map. The inclusion map $j: \Omega(\Omega X) \rightarrow L(\Omega X)$ is $\operatorname{map}_{*}(\pi, X)$. The composite $\pi \circ \sigma: S^{2} \rightarrow S^{1} \wedge S^{1}$ is the quotient map obtained by identifying a meridian with a point in the sphere $S^{2}$. The composite $\pi \circ \sigma$ can also be viewed as the quotient map from the non-reduced suspension of $S^{1}$ to the reduced suspension of $S^{1}$. So the composite $\pi \circ \sigma: S^{2} \rightarrow S^{1} \wedge S^{1}$ is a homotopy equivalence. Let $\Theta: S^{1} \wedge S^{1} \stackrel{\cong}{\longrightarrow} S^{2}$ be any given homeomorphism. 
The composite $\Theta \circ \pi \circ \sigma: S^{2} \rightarrow S^{2}$ is of degree \pm 1 . The reflection through the equatorial plane is a morphism of $S^{1}$-spaces. By replacing eventually $\sigma$ by its composite with the previous reflection, we can suppose that $\Theta \circ \pi \circ \sigma: S^{2} \rightarrow S^{2}$ is homotopic to the identity map of $S^{2}$, i.e. $\sigma \circ \Theta$ is a section of $\pi$ up to homotopy. Therefore $\operatorname{map}_{*}(\sigma \circ \Theta, X)=\operatorname{map}_{*}(\Theta, X) \circ r$ is a retract of $j$ up to homotopy.

- Let $\rho: S_{+}^{1} \wedge S^{1}=\frac{S^{1} \times S^{1}}{S^{1} \times *} \rightarrow S^{1}$ be the map induced by the projection on the second factor. Since $\pi_{2}\left(S^{1}\right)=*$, the composite $\rho \circ \sigma$ is homotopically trivial. Therefore $r \circ s$, the composite of $r=\operatorname{map}_{*}(\sigma, X)$ and $s=\operatorname{map}_{*}(\rho, X): \Omega X \rightarrow$ $L(\Omega X)$ is also homotopically trivial.

Proof of Lemma 26. Denote by $\operatorname{ad}_{S^{n}}: S^{n} \rightarrow \Omega S^{n+1}$ the adjoint of the identity map id: $S^{n+1} \rightarrow S^{n+1}$. The map $L\left(\operatorname{ad}_{S^{2}}\right): L S^{2} \rightarrow L \Omega S^{3}$ is obviously a morphism of $S^{1}$-spaces. Therefore using Proposition 28, the composite $r \circ L\left(\operatorname{ad}_{S^{2}}\right): L S^{2} \rightarrow$ $L \Omega S^{3} \rightarrow \Omega^{2} S^{3}$ is also a morphism of $S^{1}$-spaces. Therefore $H_{*}\left(r \circ L\left(\operatorname{ad}_{S^{2}}\right)\right)$ commutes with the corresponding operators $\Delta$ in $H_{*}\left(L S^{2}\right)$ and $H_{*}\left(\Omega^{2} S^{3}\right)$.

Consider the commutative diagram up to homotopy:

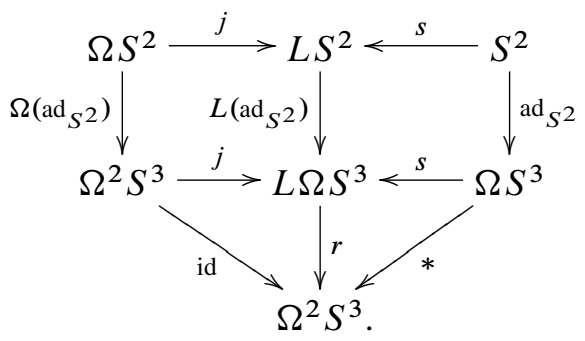

Using the left part of this diagram, we see that $\pi_{1}(r \circ L(\mathrm{ad}))$ maps the generator of $\pi_{1}\left(L S^{2}\right)=\mathbb{Z}\left(j \circ \operatorname{ad}_{S^{1}}\right)$ to the composite $\Omega\left(\operatorname{ad}_{S^{2}}\right) \circ \operatorname{ad}_{S^{1}}: S^{1} \rightarrow \Omega S^{2} \rightarrow \Omega^{2} S^{3}$ which is the generator of $\pi_{1}\left(\Omega^{2} S^{3}\right) \cong \mathbb{Z}$. Therefore $\pi_{1}(r \circ L(\mathrm{ad}))$ is an isomorphism.

So we have the commutative diagram

$$
\begin{gathered}
\pi_{1}\left(L S^{2}\right) \otimes \mathbb{F}_{2} \stackrel{\text { hur }}{\cong} H_{1}\left(L S^{2} ; \mathbb{F}_{2}\right) \stackrel{\Delta}{\longrightarrow} H_{2}\left(L S^{2} ; \mathbb{F}_{2}\right) \\
\pi_{1}\left(r \circ L\left(\operatorname{ad}_{S^{2}}\right)\right) \otimes \mathbb{F}_{2}\left|\cong H_{1}\left(r \circ L\left(\operatorname{ad}_{S^{2}}\right) ; \mathbb{F}_{2}\right)\right| \\
\pi_{1}\left(\Omega^{2} S^{3}\right) \otimes \mathbb{F}_{2} \stackrel{\text { hur }}{\cong} H_{1}\left(\Omega^{2} S^{3} ; \mathbb{F}_{2}\right) \stackrel{\Delta}{\longrightarrow} H_{2}\left(\Omega^{2} S^{3} ; \mathbb{F}_{2}\right) .
\end{gathered}
$$

By Theorem 32, $\Delta: H_{1}\left(\Omega^{2} S^{3} ; \mathbb{F}_{2}\right) \rightarrow H_{2}\left(\Omega^{2} S^{3} ; \mathbb{F}_{2}\right)$ is non-zero. Therefore using the above diagram, the composite $H_{2}\left(r \circ L\left(\operatorname{ad}_{S^{2}}\right)\right) \circ \Delta$ is also non-zero. On the other hand, using the right part of diagram (29), we have that the composite $H_{2}\left(r \circ L\left(\operatorname{ad}_{S^{2}}\right)\right) \circ H_{2}(s)$ is null. 
Corollary 30. The free loop space modulo 2 homology $\mathbb{H}_{*}\left(L S^{2} ; \mathbb{F}_{2}\right)$ is not isomorphic as Batalin-Vilkovisky algebra to the Hochschild cohomology of $H^{*}\left(S^{2} ; \mathbb{F}_{2}\right)$, $H H^{*}\left(H^{*}\left(S^{2} ; \mathbb{F}_{2}\right) ; H^{*}\left(S^{2} ; \mathbb{F}_{2}\right)\right)$.

This means exactly that there exists no isomorphism between $\mathbb{H}_{*}\left(L S^{2} ; \mathbb{F}_{2}\right)$ and $H H^{*}\left(H^{*}\left(S^{2} ; \mathbb{F}_{2}\right) ; H^{*}\left(S^{2} ; \mathbb{F}_{2}\right)\right)$ which at the same time

- is an isomorphism of algebras and

- commutes with the $\Delta$-operators,

although separately

- there exists (Corollary 23) an isomorphism of algebras between $\mathbb{H}_{*}\left(L S^{2} ; \mathbb{F}_{2}\right)$ and $H H^{*}\left(H^{*}\left(S^{2} ; \mathbb{F}_{2}\right) ; H^{*}\left(S^{2} ; \mathbb{F}_{2}\right)\right)$ and

- there exists also an isomorphism commuting with the $\Delta$-operators between them.

Proof. By Proposition 20, $H H^{*}\left(H^{*}\left(S^{2}\right) ; H^{*}\left(S^{2}\right)\right)$ is the Batalin-Vilkovisky algebra given by $\varepsilon=0$ in Lemma 21 . On the contrary, by Theorem $24, \mathbb{H}_{*}\left(L S^{2} ; \mathbb{F}_{2}\right)$ is the Batalin-Vilkovisky algebra given by $\varepsilon=1$. At the end of the proof of Lemma 21, we saw that the two cases $\varepsilon=0$ and $\varepsilon=1$ correspond to two non-isomorphic Batalin-Vilkovisky algebras.

More generally, we believe that for any prime $p$, the free loop space modulo $p$ of the complex projective space $\mathbb{H}_{*}\left(L \mathbb{C} \mathbb{P}^{p-1} ; \mathbb{F}_{p}\right)^{1}$ is not isomorphic as Batalin-Vilkovisky algebra to the Hochschild cohomology

$$
H H^{*}\left(H^{*}\left(\mathbb{C} \mathbb{P}^{p-1} ; \mathbb{F}_{p}\right) ; H^{*}\left(\mathbb{C} \mathbb{P}^{p-1} ; \mathbb{F}_{p}\right)\right)
$$

Such phenomena for formal manifolds should not appear over a field of characteristic 0 .

Recall that by Poincaré duality, we have the isomorphism (cf. Equation (19))

$$
\Theta: H^{*}\left(S^{2}\right) \stackrel{\cong}{\longrightarrow} H^{*}\left(S^{2}\right)^{\vee}
$$

Therefore we have the isomorphism

$$
H H^{*}\left(H^{*}\left(S^{2}\right) ; \Theta\right): H H^{*}\left(H^{*}\left(S^{2}\right) ; H^{*}\left(S^{2}\right)\right) \stackrel{\cong}{\longrightarrow} H H^{*}\left(H^{*}\left(S^{2}\right) ; H^{*}\left(S^{2}\right)^{\vee}\right) .
$$

Consider any isomorphism of graded algebras

$$
\mathbb{H}_{*}\left(L S^{2}\right) \cong H H^{*}\left(S^{*}\left(S^{2}\right) ; S^{*}\left(S^{2}\right)\right) .
$$

\footnotetext{
${ }^{1}$ Bökstedt and Ottosen [1] have recently announced the computation of the Batalin-Vilkovisky algebra $\mathbb{H}_{*}\left(L \mathbb{C} \mathbb{P}^{n} ; \mathbb{F}_{p}\right)$.
} 
By Corollary 23, such isomorphism exists. Cohen and Jones ([7, Theorem 3] and [5]) proved that such isomorphism exists for any manifold $M$. Since $S^{2}$ is formal, we have the isomorphism of algebras (cf. Equation (2))

$$
H H^{*}\left(S^{*}\left(S^{2}\right) ; S^{*}\left(S^{2}\right)\right) \stackrel{\cong}{\longrightarrow} H H^{*}\left(H^{*}\left(S^{2}\right) ; H^{*}\left(S^{2}\right)\right) .
$$

By [17], we have the isomorphisms of $H_{*}\left(S^{1}\right)$-modules

$$
H_{*}\left(L S^{2}\right) \stackrel{(14)}{\cong} H H^{*}\left(S^{*}\left(S^{2}\right) ; S^{*}\left(S^{2}\right)^{\vee}\right) \stackrel{(15)}{\cong} H H^{*}\left(H^{*}\left(S^{2}\right) ; H^{*}\left(S^{2}\right)^{\vee}\right) .
$$

Corollary 30 implies that the following diagram does not commute over $\mathbb{F}_{2}$ :

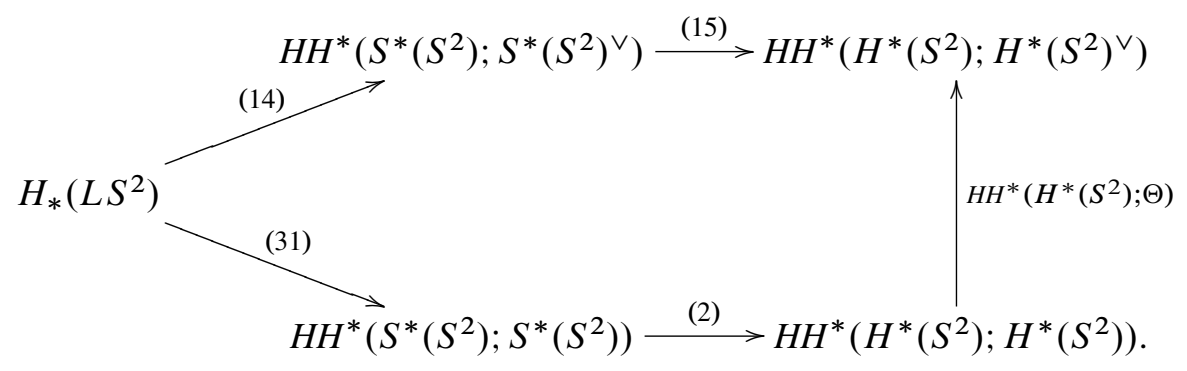

This is surprising because as explained by Cohen and Jones [7, p. 792], the composite of the isomorphism (14) given by Jones in [17] and an isomorphism induced by Poincaré duality should give an isomorphism of algebras between $\mathbb{H}_{*}\left(L S^{2}\right)$ and $H H^{*}\left(S^{*}\left(S^{2}\right) ; S^{*}\left(S^{2}\right)\right)$.

\section{Appendix by Gerald Gaudens and Luc Menichi}

Let $X$ be a pointed topological space. Recall that the circle $S^{1}$ acts on the double loop space $\Omega^{2} X$. Consider the induced operator $\Delta: H_{*}\left(\Omega^{2} X\right) \rightarrow H_{*+1}\left(\Omega^{2} X\right)$. Getzler [14] has shown that $H_{*}\left(\Omega^{2} X\right)$ equipped with the Pontryagin product and this operator $\Delta$ forms a Batalin-Vilkovisky algebra. In [12], Gerald Gaudens and the author have determined this Batalin-Vilkovisky algebra $H_{*}\left(\Omega^{2} S^{3} ; \mathbb{F}_{2}\right)$. The key was the following theorem. In [18, Proposition 7.46], answering to a question of Gerald Gaudens, Sadok Kallel and Paolo Salvatore give another proof of this theorem.

Theorem 32 ([12]). The operator $\Delta: H_{1}\left(\Omega^{2} S^{3} ; \mathbb{F}_{2}\right) \rightarrow H_{2}\left(\Omega^{2} S^{3} ; \mathbb{F}_{2}\right)$ is non-trivial.

Both proofs [12] and [18, Proposition 7.46] are unpublished and publicly unavailable yet. So the goal of this section is to give a proof of this theorem which is as simple as possible. 
Denote by $*$ the Pontryagin product in $H_{*}\left(\Omega^{2} X\right)$ and by $\circ$ the map induced in homology by the composition map $\Omega^{2} X \times \Omega^{2} S^{2} \rightarrow \Omega^{2} X$. Denote by $\Omega_{n}^{2} S^{2}$, the path-connected component of the degree $n$ maps. Denote by $v_{1}$ the generator of $H_{1}\left(\Omega_{0}^{2} S^{2} ; \mathbb{F}_{2}\right)$ and by [1] the generator of $H_{0}\left(\Omega_{1}^{2} S^{2} ; \mathbb{F}_{2}\right)$.

Lemma 33. For $x \in H_{*}\left(\Omega^{2} X ; \mathbb{F}_{2}\right), \Delta x=x \circ\left(v_{1} *[1]\right)$.

Proof. The circle $S^{1}$ acts on the sphere $S^{2}$. Therefore we have a morphism of topological monoids $\Theta:\left(S^{1}, 1\right) \rightarrow\left(\Omega_{1}^{2} S^{2}, \mathrm{id}_{S^{2}}\right)$. The action of $S^{1}$ on $\Omega^{2} X$ is the composite $S^{1} \times \Omega^{2} X \stackrel{\Theta \times \Omega^{2} X}{\longrightarrow} \Omega_{1}^{2} S^{2} \times \Omega^{2} X \stackrel{\circ}{\longrightarrow} \Omega^{2} X$. Therefore for $x \in$ $H_{*}\left(\Omega^{2} X ; \mathbb{F}_{2}\right), \Delta x=x \circ\left(H_{1}(\Theta)\left[S^{1}\right]\right)$.

Suppose that $H_{1}(\Theta)\left[S^{1}\right]=0$. Then for any topological space $X$, the operator $\Delta$ on $H_{*}\left(\Omega^{2} X ; \mathbb{F}_{2}\right)$ is null. Therefore, for any $x$ and $y \in H_{*}\left(\Omega^{2} X ; \mathbb{F}_{2}\right),\{x, y\}=$ $\Delta(x y)-(\Delta x) y-x(\Delta y)=0$. That is the modulo 2 Browder brackets on any double loop space are null. This is obviously false. For example, Cohen in [3] explains that the Gerstenhaber algebra $H_{*}\left(\Omega^{2} \Sigma^{2} Y\right)$ has in general many non-trivial Browder brackets. So the assumption $H_{1}(\Theta)\left[S^{1}\right]=0$ is false.

Since the loop multiplication by id $S^{2}$ in the $H$-group $\Omega^{2} S^{2}$ is a homotopy equivalence, the Pontryagin product by [1], *[1]: $H_{*}\left(\Omega_{0}^{2} S^{2}\right) \stackrel{\cong}{\longrightarrow} H_{*}\left(\Omega_{1}^{2} S^{2}\right)$ is an isomorphism. Therefore $v_{1} *[1]$ is a generator of $H_{1}\left(\Omega_{1}^{2} S^{2}\right)$, hence $H_{1}(\Theta)\left[S^{1}\right]=v_{1} *[1]$. So finally

$$
\Delta x=x \circ\left(H_{1}(\Theta)\left[S^{1}\right]\right)=x \circ\left(v_{1} *[1]\right) .
$$

Recall that $v_{1}$ denotes the generator of $H_{1}\left(\Omega_{0}^{2} S^{2} ; \mathbb{F}_{2}\right)$.

Lemma 34. In the Batalin-Vilkovisky algebra $H_{*}\left(\Omega^{2} S^{2} ; \mathbb{F}_{2}\right), \Delta\left(v_{1}\right)=v_{1} * v_{1}$.

Proof. Recall that [1] is the generator of $H_{0}\left(\Omega_{1}^{2} S^{2}\right)$. By Lemma 33,

$$
\Delta[1]=[1] \circ\left(v_{1} *[1]\right)=\left(v_{1} *[1]\right) .
$$

Denote by $Q: H_{q}\left(\Omega_{n}^{2} S^{2}\right) \rightarrow H_{2 q+1}\left(\Omega_{2 n}^{2} S^{2}\right)$ the Dyer-Lashof operation. It is well known that $Q[1]=v_{1} *$ [2]. So by [4, p. 218, Theorem 1.3 (4)]

$$
\left\{v_{1} *[2],[1]\right\}=\{Q[1],[1]\}=\{[1],\{[1],[1]\}\} .
$$

By $[4$, p. 215, Theorem $1.2(3)],\{[1],[1]\}=0$. Therefore on one hand, $\left\{v_{1} *[2],[1]\right\}$ is null. And on the other hand, using the Poisson relation (7), since $\{[2],[1]\}=$ $\{[1] *[1],[1]\}=2\{[1],[1]\} *[1]=0$,

$$
\left\{v_{1} *[2],[1]\right\}=\left\{v_{1},[1]\right\} *[2]+v_{1} *\{[2],[1]\}=\left\{v_{1},[1]\right\} *[2] .
$$


Since $*[1]: H_{*}\left(\Omega^{2} S^{2}\right) \stackrel{\cong}{\longrightarrow} H_{*}\left(\Omega^{2} S^{2}\right)$ is an isomorphism, we obtain that the Browder bracket $\left\{v_{1},[1]\right\}$ is null. Therefore,

$$
\Delta\left(v_{1} *[1]\right)=\left(\Delta v_{1}\right) *[1]+v_{1} *(\Delta[1])=\left(\left(\Delta v_{1}\right)-v_{1} * v_{1}\right) *[1] .
$$

But $\Delta\left(v_{1} *[1]\right)=(\Delta \circ \Delta)([1])=0$. Therefore $\left(\Delta v_{1}\right)$ must be equal to $v_{1} * v_{1}$.

Proof of Theorem 32. We remark that since $\Delta$ preserves path-connected components and since the loop multiplication of two homotopically trivial loops is a homotopically trivial loop, $H_{*}\left(\Omega_{0}^{2} S^{2}\right)$ is a sub Batalin-Vilkovisky algebra of $H_{*}\left(\Omega^{2} S^{2}\right)$.

Let $S^{1} \hookrightarrow S^{3} \stackrel{\eta}{\longrightarrow} S^{2}$ be the Hopf fibration. After double looping, the Hopf fibration gives the fibration $\Omega^{2} S^{1} \hookrightarrow \Omega^{2} S^{3} \stackrel{\Omega^{2} \eta}{\longrightarrow} \Omega_{0}^{2} S^{2}$ with contractile fiber $\Omega^{2} S^{1}$ and path-connected base $\Omega_{0}^{2} S^{2}$. Therefore $\Omega^{2} \eta: \Omega^{2} S^{3} \stackrel{\simeq}{\longrightarrow} \Omega_{0}^{2} S^{2}$ is a homotopy equivalence. And so $H_{*}\left(\Omega^{2} \eta\right): H_{*}\left(\Omega^{2} S^{3}\right) \stackrel{\simeq}{\longrightarrow} H_{*}\left(\Omega_{0}^{2} S^{2}\right)$ is an isomorphism of Batalin-Vilkovisky algebras.

Let $u_{1}$ be the generator of $H_{1}\left(\Omega^{2} S^{3}\right)$. Lemma 34 implies that $\Delta\left(u_{1}\right)=u_{1} * u_{1}$. Since $u_{1} * u_{1}$ is non-zero in $H_{*}\left(\Omega^{2} S^{3} ; \mathbb{F}_{2}\right), \Delta\left(u_{1}\right)$ is non-trivial.

\section{References}

[1] M. Bökstedt and I. Ottosen, The homology of the free loop space on a projective space. Talk at the First Copenhagen Topology Conference, September 1-3, 2006; http://www.math.ku.dk/conf/CTC2006

[2] M. Chas and D. Sullivan, String topology. Preprint 1999, arXiv:math/9911159

[3] F. Cohen, On configuration spaces, their homology, and lie algebras. J. Pure Appl. Algebra 100 (1-3) (1995), 19-42. Zbl 0921.57011 MR 1344842

[4] F. Cohen, T. Lada, and J. May, The homology of iterated loop spaces. Lecture Notes in Math. 533, Springer-Verlag, Berlin 1976. Zbl 0334.55009 MR 0436146

[5] R. Cohen, Multiplicative properties of Atiyah duality. Homology Homotopy Appl. 6 (1) (2004), 269-281. Zbl 1072.55004 MR 2076004

[6] R. Cohen, K. Hess, and A. Voronov, String topology and cyclic homology. Lectures from the Summer School held in Almería, 2003 Adv. Courses Math. CRM Barcelona, Birkhäuser, Basel 2006. Zbl 1089.57002 MR 2251006

[7] R. Cohen and J. Jones, A homotopic theoretic realization of string topology. Math. Ann. 324 (4) (2002), 773-798. Zbl 1025.55005 MR 1942249

[8] R. Cohen, J. Jones, and J. Yan, The loop homology algebra of spheres and projective spaces. In Categorical decomposition techniques in algebraic topology (Isle of Skye 2001), Prog. Math. 215, Birkhäuser, Basel 2004, 77-92. Zbl 1054.55006 MR 2039760

[9] K. Costello, Topological conformal field theories and Calabi-Yau categories. Adv. Math. 210 (1) (2007), 165-214. Zbl 05132553 MR 2298823 
[10] Y. Félix, L. Menichi, and J.-C. Thomas, Gerstenhaber duality in Hochschild cohomology. J. Pure Appl. Algebra 199 (1-3) (2005), 43-59. Zbl 1076.55003 MR 2134291

[11] Y. Félix, J.-C. Thomas, and M. Vigué-Poirrier, Rational string topology. J. Eur. Math. Soc. 9 (1) (2005), 123-156. Zbl 05129007 MR 2283106

[12] G. Gaudens and L. Menichi, Batalin-Vilkovisky algebras and the J-homomorphism. Topology Appl. 156 (2) (2008), 365-374.

[13] M. Gerstenhaber, The cohomology structure of an associative ring. Ann. of Math. 78 (2) (1963), 267-288. Zbl 0131.27302 MR 0161898

[14] E. Getzler, Batalin-Vilkovisky algebras and two-dimensional topological field theories. Comm. Math. Phys. 159 (2) (1994), 265-285. Zbl 0131.27302 MR 0161898

[15] K. Gruher and P. Salvatore, Generalized string topology operations. Proc. Lond. Math. Soc. (3) 96 (1) (2008), 78-106. Zbl 1143.57012 MR 2392316

[16] A. Hatcher, Algebraic topology. Cambridge University Press, Cambridge 2002. Zbl 1044.55001 MR 1867354

[17] J. D. S. Jones, Cyclic homology and equivariant homology. Invent. Math. 87 (2) (1987), 403-423. Zbl 1012.20002 MR 0870737

[18] S. Kallel, Loop spaces, symmetric products and configuration spaces. Book in preparation; previously available at http://math.univ-lille1.fr/ kallel

[19] R. Kaufmann, A proof of a cyclic version of Deligne's conjecture via Cacti. Math. Res. Letters 15 (5) (2008), 901-921. MR 2443991

[20] — Moduli space actions on the Hochschild co-chains of a Frobenius algebra I:Cells operads. J. Noncommut. Geom. 1 (3) (2007), 333-384. Zbl 1145.55008 MR 2314100

[21] M. Kontsevich and Y. Soibelman, Notes on A-infinity algebras, A-infinity categories and non-commutative geometry. I. Preprint 2006, arXiv:math.RA/0606241

[22] L. Menichi, Batalin-Vilkovisky algebras and cyclic cohomology of Hopf algebras. $K$ Theory 32 (3) (2004), 231-251. Zbl 1101.19003 MR 2114167

[23] S. Merkulov, De Rham model for string topology. Internat. Math. Res. Notices 2004 (55) (2004), 2955-2981. Zbl 1066.55008 MR 2099178

[24] A. Stacey, The differential topology of loop spaces. Preprint 2005, arXiv:math.DG/0510097

[25] H. Tamanoi, Batalin-Vilkovisky Lie algebra structure on the loop homology of complex Stiefel manifolds. Internat. Math. Res. Notices 2006 (2006), 1-23. Zbl 1108.55007 MR 2211159

[26] T. Tradler, The BV algebra on Hochschild cohomology induced by infinity inner products. Ann. Inst. Fourier, to appear; Preprint 2002, arXiv:math.QA/0210150

[27] T. Tradler and M. Zeinalian, On the cyclic Deligne conjecture. J. Pure Appl. Algebra 204 (2) (2006), 280-299. Zbl 02242211 MR 2184812

[28] D. Vaintrob, The string topology BV algebra, Hochschild cohomology and the Goldman bracket on surfaces. Preprint 2007, arXiv:math/0702859

[29] M. Vigué-Poirrier, Decomposition de l'homologie cyclique des algèbres différentielles graduées commutatives. K-Theory 4 (5) (1991), 399-410. Zbl 0731.19004 MR 1116926 
[30] C. Westerland, Dyer-Lashof operations in the string topology of spheres and projective spaces. Math. Z. 250 (3) (2005), 711-727. Zbl 1076.55006 MR 2179618

[31] - String homology of spheres and projective spaces. Algebr. Geom. Topol. 7 (2007), 309-325. Zbl 05220877 MR 2308947

Received March 15, 2007

Luc Menichi, UMR 6093 associée au CNRS, Université d'Angers, Faculté des Sciences, 2 Boulevard Lavoisier, 49045 Angers, France

E-mail: luc.menichi@univ-angers.fr 